\title{
COGNITIVE, INTELLIGENCE TECHNOLOGIES AND ECONOMIC FOUNDATIONS OF TEACHING OF INTERNATIONAL ECONOMIC RELATIONS AND TOURISM
}

\author{
Mariana Petrova ${ }^{1}$, Iryna Buzko ${ }^{2}$, Yuriy Dyachenko ${ }^{2}$ \\ ${ }^{1}$ St. Cyril and St. Methodius University of Veliko Tarnovo, Bulgaria; \\ ${ }^{2}$ Volodymyr Dahl East Ukrainian National University, Ukraine \\ m.petrova@uni-vt.bg,meo@snu.edu.ua,yuriy.y.dyachenko@gmail.com
}

\begin{abstract}
As an approach to foresee future changes in workforce marketplace, we propose upgraded curricula with emphasis on acquiring of intelligent and cognitive technologies. To achieve the possibilities of cognitive and intelligence technologies by students we developed the curriculum "International Economic Relations" in compliance with the employers' demands. We consider management of education as a process aiming to improve the structure of both educational services and knowledge. Based on the epistemological position of critical realism we develop the prescriptive methodology of education as a cyclic process and its description that allows systematizing internal components and external influencers in the educational process. To find the economic effects of education we strike with simplified and isolated conditions of economic models that usually cannot be reproduced experimentally, making it difficult to verify the truth of empirical economic theories. We use intelligent systems that allow the automation of student-teacher communication due to providing the interaction between a teacher, a student and an educational institution. As an example of artificial intelligence technologies used for management of education, we consider determining the impact of performance of the company on indicators characterizing the personnel training. This analysis is performed on the basis of cognitive system IBM Watson Analytics.
\end{abstract}

Keywords: cognitive technologies, international economic relations, tourism.

\section{Introduction}

We face the technological-driven openness in international economic relations in the conditions of globalization and information technolgies development due to needs for Big Data, increasing investments in research and development and international influence of national regulations in the sphere of information security and ethics. Education, particularly, in the field of international economic relations and tourism under these circumstances comes with some challenges such as high competitiveness, growing risks, lack of predictability $[1 ; 2]$.

Economical approach is based on scarcity of resources and following optimization of resource allocation and consumption. One of the ways to solve this problem is exploring of intelligent and cognitive technologies. For example, Google's DeepMind research [3] has explored the problem of resource allocation using artificial intelligence methods. In economic thinking people are concerned with accounting for asymmetric information (certain agents have more information about the market than others), causality or endogeneity (which factors are influencing the output internally within the workings of the model system), or multi-collinearity (dependencies with the multivariate independent variables that may lead to spurious correlations and unreliable model forecasts) [4].

Due to technological innovations, international economical relations will undergo the following significant changes [5].

1. International economical relations will be largely determined by massive global platforms. Governments will be seeking to reign in their power through regulation or legislation. But corporations will accelerate their efforts to secure the platform advantage and raise the baseline, from which their settlement will be judged in the years to come.

2. National AI strategies will emerge from more countries for more grounds for co-operation and more reasons to argue about intellectual property, privacy, data and license to operate.

3. Silicon Valley's political culture will be disseminated into software, corporate culture and strategy. Particularly, crypto whizzes will establish governance mechanisms on emerging blockchain networks. This matters because information technology systems affect how we perceive our set of choices and act in that world.

Economic systems are embedded with colossal number of dynamic components including agents, institutions, and constructs that vary over time. These dependencies are not well understood. And cognitive, intelligence technologies could be useful for such highly complex tasks [4]. Economic theory suggests that artificial intelligence will raise the value of human judgement, as predictions 
becoming cheaper; human beings will have the opportunity to exercise greater weighting of costs and benefits to make better judgement [6]. These structural changes in computational capacity, large-scale data and deep learning will not exclude human beings, but perhaps give power to high level decision makers strengthening pre-existing norms and organizational culture [4].

Education, particularly, in the field of International Economic Relations and Tourism in the modern age strikes some challenges such as high competitiveness, growing risks, lack of predictability. We consider management of education as a process aiming to improve the structure of providers of educational services and educational structure of society. For construction of this structure we need to consider some basic assumptions. Foundation of any science is its ontology as description of nature and categories of the subject area.

\section{Materials and methods}

One of the most influential ontological doctrines is realism that asserts the reality of certain objects, depending on the type there are several options of ontological realism: universals or ideas Platonic or Aristotelian in realism, scientific objects of scientific realism, etc. [7]. This fundamental, ontological meaning of realism may be supplemented by an epistemological point of view, according to which real objects are also recognizable. This epistemological realism asserts that the facts about these objects are known or can be known, that the subject of knowledge has epistemological access to the object of knowledge and there is no insurmountable barrier between the perceiving the subject and the existing object [7]. Economic realism argues that economic reality is an objective (although to some extent dependent on consciousness) existing structure, and economic theories, albeit incomplete and some false elements capable to reflect some important aspects of reality correctly [7].

Critical realism, whereby the world that is the subject of scientific research, consists not only of events given to us in experience or perception, but also (those which are not limited to them) of structures, machinery, driving forces and trends, which, although not directly observable, but nevertheless underlying real events, manage them or facilitate their implementation [8]. T. Lawson proved the theory of social ontology, according to which various forms of social structures (relationships, rules, institutions) form a relatively autonomous area, which is the result of interaction between humans and dependent on it, but has properties that cannot be reduced to this interaction and it has the opposite effect [8]. Within this paradigm the world consists of the objects that are structured and structured not transitive, that is, first, not confined to events (which may be present or may not be present in our experience) and secondly, exist independently of their identification [8]. Thus, there are three fields of reality: empirical (experience and perception), non-empirical really existing (development and status quo) and "deep" (structures, mechanisms and driving forces and trends), and these three areas are not reducible to each other and not "synchronous", i.e. not experience coincides with events, allowing us to compare and browse the perception of a phenomenon, and events do not coincide with those mechanisms that control them identify [8]. The purpose of the economic theory is identifying structures that govern these activities, the conditions in which it is immersed, which facilitate it and are transformed through it, these explanations entail understanding certain practices, analysis of unknown conditions for their implementation, unconscious motivation behind them, imply the skills used in these practices and their unintended consequences [8]. Despite the fact that society and the economy are the product of economic agents, they are opaque to some extent for these agents, so the task of the economic theory is the description of all that has to be done (or not that aware of the processes involved in agent) to a isolated social phenomenon, a set of practices or actions made possible [8].

Based on the definition of the methodology as a field of knowledge, establishing a system of concepts and their correlation in a form of scientific practice, research activities in methodology can be seen in the light of the scientific value of truth and non-scientific elements and separation the first from the second (normative or prescriptive methodology) [9].

\section{Results and Discussion}

While considering distance learning in education, cognitive technologies for Human Resource development and artificial technologies in education we focus on methodology of education [10]. The greatest advantage of the intelligent and cognitive technologies is the ability to provide an individual 
approach to students given the opportunity to learn the language at an individual pace and sequence that takes into account the psychological characteristics of each student, which greatly improves the quality of mastering linguistic material. As an approach to foresee future changes at workplace we propose upgraded curricula with emphasis on intelligent and cognitive technologies.

The use of intelligent systems allows the automation of student-teacher communication due to providing the interaction between a teacher, a student and an educational institution. The proposed variant of the architecture of such a system used Facebook platform with Artificial Intelligence powered with AI chatbot. For the students this enables to integrate learning in familiar for student social network environment. As for the teachers this enables to reduce the burden and to relieve them from monotonous work. The educational institution will be able to organize the learning in a more flexible way. Also the proposed approach could be one of the first steps towards machine teaching. Transition from discrete paradigm of information processing (programming for Turing machines) to continuous paradigm (learning of artificial intelligence) allows faster and more accurate adaptation to environmental requirements. In future it can yield the most suitable solution for knowledge transfer from one agent to another in order to ensure inclusive and equitable quality education.

Based on the above epistemological position of critical realism let us consider the structure of the normative methodology of education.

The position of critical realism supporters, epistemological knowledge structure consists of real, actual and empirical. Real exists itself, regardless of the subject, knowing and availability of real objects for observation; empirical - this is the reality as it is presented in the experience and observation; actual - is the intermediate level, which corresponds to reality as orderly sequence of events or conditions [8].

Prescriptive methodology of education is a cyclic process and its description allows to systematize the components of training and define the epistemological status characteristics, which determine the educational process (Fig. 1). The content of education depends on the subject field and its modern state and perspectives.

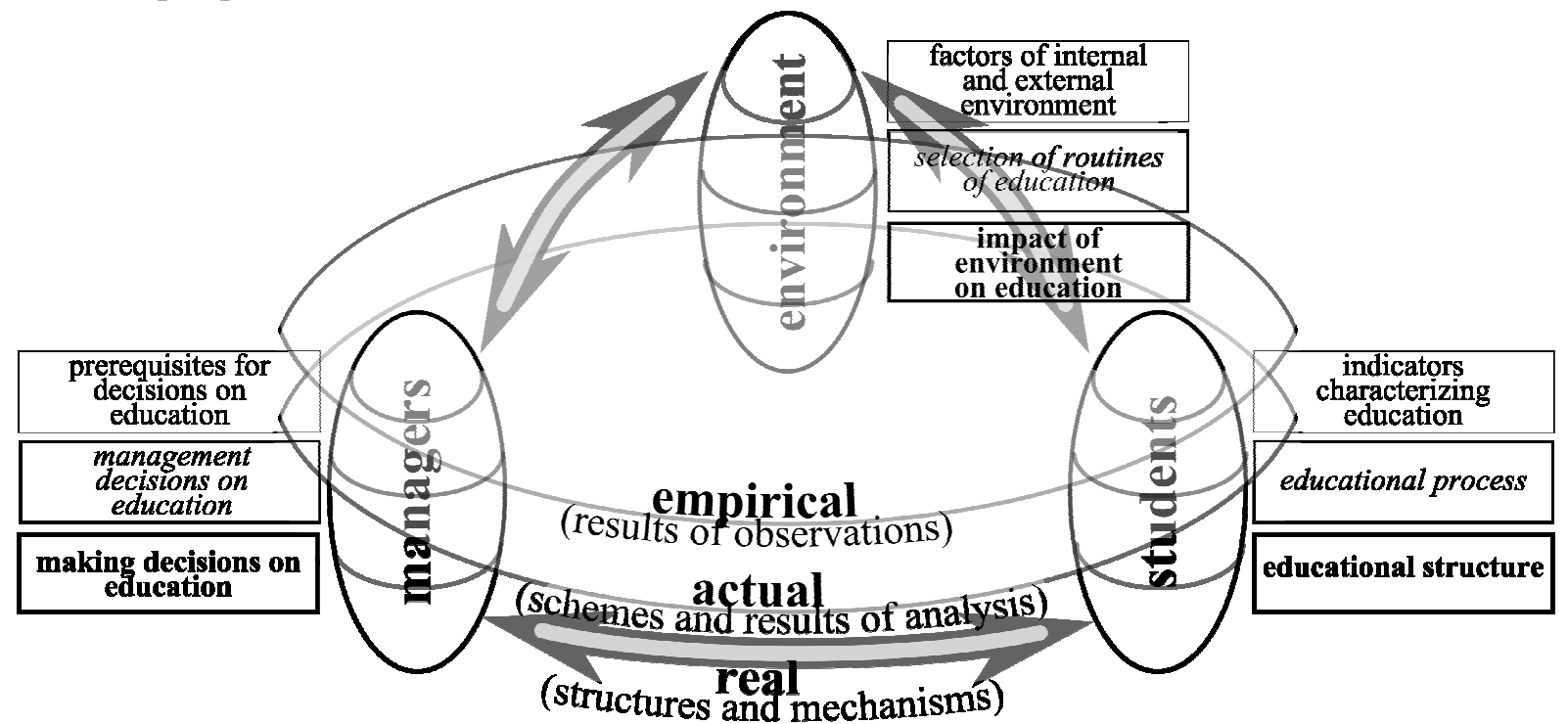

Fig. 1. Prescriptive methodology of education

We use intelligent systems that allow automation of student-teacher communication due to providing the interaction between a teacher, a student and an educational institution.

Now Big Data and new machine learning techniques could help test the relative power of competing theories with a better sense of what is influencing behaviour in the economy [11]. Also economists will treat "model is a model, not the model" [11].

At the same time simplified and isolated conditions of economic models usually cannot be reproduced experimentally, making it difficult to verify the truth of empirical economic theories and therefore economic realists are fallibilsts taking non-final character existing knowledge and the ability to adjust and review in the light of new evidence and provements [7]. 
As an example of Artificial Intelligence used for management of education, we consider determining the impact of performance of the company (labour costs, labour costs per person, income, profit, profit per person) on indicators characterizing the personnel training, the analysis of this relationship for ALC "SZKhNO” [12] (Table 1).

Table 1

Financial results, labour costs and share of trained persons in ALC “SZKhNO" in 2007-2014

\begin{tabular}{|c|c|c|c|c|c|c|c|c|}
\hline Index & 2007 & 2008 & 2009 & 2010 & 2011 & 2012 & 2013 & 2014 \\
\hline Persons & 231 & 274 & 272 & 272 & 273 & 314 & 321 & 286 \\
\hline Labour costs, ths. UAH & 4254.0 & 5849.6 & 2718.2 & 5044.5 & 6078.6 & 7109.6 & 6849.8 & 3640.0 \\
\hline $\begin{array}{l}\text { Labour costs per person, } \\
\text { ths. UAH per person }\end{array}$ & 18.42 & 21.35 & 9.99 & 18.55 & 22.27 & 22.64 & 21.34 & 12.73 \\
\hline Income, ths. UAH & 25740 & 49752 & 46250 & 47233 & 76628 & 70137 & 96649 & 98151 \\
\hline Profit, ths. UAH & -959 & 6022 & 1823 & -6825 & 5711 & 7036 & 10766 & 14098 \\
\hline $\begin{array}{l}\text { Profit per person, ths. } \\
\text { UAH per person }\end{array}$ & -4.15 & 21.98 & 6.70 & -25.09 & 20.92 & 22.41 & 33.54 & 49.29 \\
\hline $\begin{array}{l}\text { Number of trained } \\
\text { persons }\end{array}$ & 49 & 46 & 36 & 25 & 43 & 42 & 21 & 14 \\
\hline $\begin{array}{l}\text { Share of trained } \\
\text { persons, } \%\end{array}$ & 21.2 & 16.8 & 13.2 & 9.2 & 15.8 & 13.4 & 6.5 & 5.0 \\
\hline
\end{tabular}

This analysis [12] is performed on the basis of cognitive system IBM Watson Analytics (Figure 2). This is a technology platform that uses natural language processing and machine learning. IBM Watson Analytics is an easy-to-use service for finding answers in data without downloading software. Discovery of visualization and smart solution available on the cloud, it guides data exploration, automated predictive analytics and makes creating dashboards and infographics almost effortless.

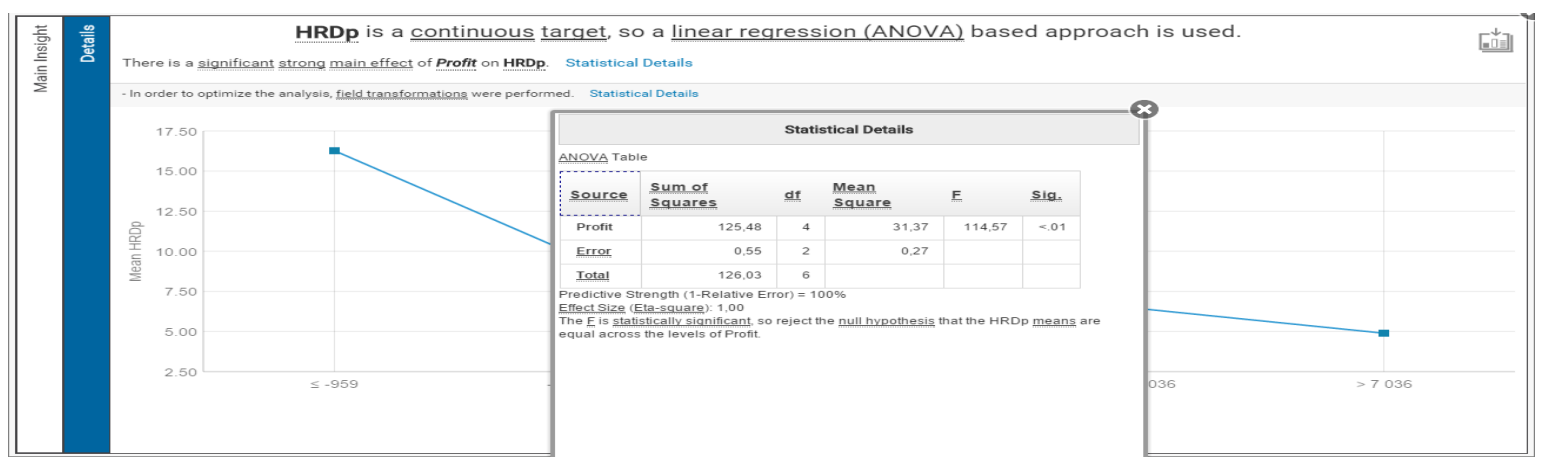

Fig. 2. Results of IBM Watson Analytics analysis of data set

It was found that the main factor influencing the amount of training the company is the net income of the company for the previous year. Considering this analysis it could be argued that decisions on financing of education are carried out on the basis of income of the enterprise in the past period.

We have been working under the assumption that main demand for competencies of students of "International Economic Relations" and "Tourism" lies in development of cognitive, communicative and technological abilities.

To achieve the possibilities of cognitive and intelligence technologies by students we developed the curriculum "International Economic Relations" witch specialization "International Business and Cognitive Economics" based on relevant stakeholders. We include disciplines "Information management", "Intelligent Technology in Corporate Governance", "Cognitive Technologies in Economics". 


\section{Conclusions}

Methodology of education describes education as a cyclical process and its description enables to systematize the components of training and define of the epistemological status of an educational process. On this basis, we propose to apply cognitive technologies for solving of highly complex tasks as automation of student-teacher communication and calculation of educational effectiveness. As an example of Artificial Intelligence technologies used for management of education, we consider determining the impact of performance of the company on the indicators characterizing the personnel training. It was found that the main factor influencing the amount of training in the company is the net income of the company for the previous year. Considering this analysis it could be argued that decisions on financing of education are carried out on the basis of income of the enterprise in the past period. To familiarize "International Economic Relations" and "Tourism" students with such technologies we develop the curriculum "International Economic Relations" with specialization "International Business and Cognitive Economics" based on relevant stakeholders.

\section{Acknowledgements}

The research is financed by Project № FSD - 31-813/ 28.06.2016 „Developing a web portal of the electronic periodicals of "St. Cyril and St. Methodius" University of Veliko Tarnovo, Bulgaria.

\section{References}

[1] Nenkov N., Sushchenko O., Dyachenko Y. Role of chief information officer within the system of human resource development in service organizations (tourism). Economic Annals-XXI, 2017, Volume 165, Issue 5-6, pp. 97-103.

[2] Nenkov N., Petrova M., Dyachenko Y. Bondarenko G., Pustovit V. Intelligent and Cognitive Technologies in Education of International Economic Relations Students and Human Resource Development: Methodology in Language Teaching and Distance Learning. European Journal of Sustainable Development, 2017, Vol. 6, No. 4, pp. 353-360.

[3] Perolat J., Leibo J.Z., Zambaldi V., Beattie C., Tuyls K., Graep T. et al. A multi-agent reinforcement learning model of common-pool resource appropriation. Advances in Neural Information Processing Systems, 2017, pp. 3645-3654

[4] Perez C.E. Automating High-Level Economic Thinking using Deep Learning. Medium, 2017, December 16, https://medium.com/intuitionmachine/economic-modeling-and-deep-learningdcd61b351cad

[5] Azhar A. Exponential View, 2017, vol. 146. [online][17.01.2018] Available at: http://mailchi.mp/exponentialview/ev146? $=187056 \mathrm{a} 5 \mathrm{c} 9$

[6] Agrawal A., Gans J., Goldfarb A. How AI will change the way we make decisions. Harvard Business Review, 2017, July 26, [online][17.01.2018] Available at: https://hbr.org/2017/07/howai-will-change-the-way-we-make-decisions

[7] Mäki U. Realism. The Handbook of Economic Methodology. Cheltenham: Edward Elgar, 1998. pp. 404-409.

[8] Lawson T. A Realist Perspective on Contemorary "Economic Theory". Journal of Economic Issues, 1995, Vol. 29, No. 1, pp. 1-32.

[9] Болдырев И.А. Экономическая методология сегодня: краткий обзор основных направлений. Журнал Новой экономической ассоциации (Boldyrev I.A. Economic methodology today: a brief overview of the main directions. Journal of the New Economic Association), 2011, vol. 9, pp. 47-70. (In Russian).

[10] Nenkov N., Dyachenko Y., Dimitrov G., Koeva K. Artificial Intelligence Technologies for Personnel Learning Management Systems. 2016 IEEE 8th International Conference on Intelligent Systems. Proceedings, Sofia, Bulgaria, 2016, pp. 189-194.

[11] A less dismal science, Economist, vol. 420, 2016, p. 12.

[12] Buzko I., Dyachenko Y., Petrova M., Nenkov N., Tuleninova D., Koeva K. Artificial Intelligence Technologies in Human Resource Development // Computer Modelling and New Technologies. 2016. Vol. 20. № 2. pp. 26-29. 